\title{
A Patient with Spinal Arteriovenous Malformation Treated with Lumbar Vertebral Surgery Due to Misdiagnosis
}

\author{
Shuhei Matsuoka*, Shintaro Tsuge, Kazumasa Nakamura, Keiji Hasegawa, Akihito Wada, \\ Hiroshi Takahashi \\ Department of Orthopaedic Surgery, Toho University School of Medicine, Tokyo, Japan \\ Email: *shuhei.matsuoka@med.toho-u.ac.jp
}

How to cite this paper: Matsuoka, S., Tsuge, S., Nakamura, K., Hasegawa, K., Wada, A. and Takahashi, H. (2020) A Patient with Spinal Arteriovenous Malformation Treated with Lumbar Vertebral Surgery Due to Misdiagnosis. Open Journal of Orthopedics, 10, 193-202.

https://doi.org/10.4236/ojo.2020.109022

Received: July 26, 2020

Accepted: August 30, 2020

Published: September 2, 2020

Copyright $\odot 2020$ by author(s) and Scientific Research Publishing Inc. This work is licensed under the Creative Commons Attribution International License (CC BY 4.0).

http://creativecommons.org/licenses/by/4.0/

\begin{abstract}
Spinal arteriovenous malformation (AVM) is a rare disease that arises more commonly from the lower thoracic and upper lumbar spine. It is classified based on the development pattern and the shunt location. The developmental mechanism of the symptoms is thought to be as follows. Spinal venous return is impaired by high-pressure arterial blood flowing into the coronary sinus via a shunt and venous pressure is promoted, which causes spinal cord symptoms to progress gradually. Listlessness, pain, and an abnormal sensation of the lower limb are possible initial symptoms. Spinal AVM may cause intermittent claudication and bladder and rectal disturbance, and differentiation from lumbar degenerative diseases and arteriosclerosis obliterans is required, which may cause difficulty with diagnosis. We encountered a patient in whom intermittent claudication was treated with lumbar decompression and fixation at another hospital, but symptoms did not improve. The patient was diagnosed with spinal AVM at our hospital and symptoms were improved by surgery. Because the symptoms did not improve despite being treated with surgery for spinal canal stenosis, we strongly suspected spinal intermittent claudication, and we performed a spinal CT and MRI after myelography for the entire spinal cord, and identified the lesion in the thoracic spinal cord. Regarding the postoperative outcome and prognosis of spinal dural AVF, early diagnosis and early treatment have been proposed as prognostic factors because the postoperative outcome is poor in patients with high preoperative severity and a long duration of illness. In our patient, the preoperative JOA score was 6 , showing high preoperative severity, and the duration of illness was 1.5 years before diagnosis, which may explain the limited improvement of the JOA score to 20 at final follow-up. In a case with these characteristics, we suggest that close examination of the entire spinal cord is needed for effective treatment.
\end{abstract}




\section{Keywords}

Spinal Arteriovenous Malformation, Intermittent Claudication

\section{Introduction}

Spinal arteriovenous malformation (AVM) is a rare disease that arises more commonly from the lower thoracic and upper lumbar spine. It is classified based on the development pattern and the shunt location.

Symptoms include low back pain, progressive numbness and listlessness of the lower limb, intermittent claudication, and occasionally bladder and rectal disturbance. The developmental mechanism of the symptoms is thought to be as follows. The spinal venous return is impaired by high-pressure arterial blood flowing into the coronary sinus via a shunt and venous pressure is promoted, which causes spinal cord symptoms to progress gradually.

Cases with intermittent claudication require differentiation from lumbar spinal canal stenosis and arteriosclerosis obliterans, and the disease may be difficult to diagnose. We encountered a patient in whom intermittent claudication was treated with lumbar decompression and fixation at another hospital, but symptoms did not improve. The patient was diagnosed with spinal AVM at our hospital and symptoms were improved by surgery.

\section{Case Report}

The patient was a 73-year-old male with a chief complaint of gait disorder. $\mathrm{He}$ had become aware of lumbago about 10 years earlier, which manifested as a dull pain. He especially felt pain upon waking up, and the pain improved after movement for about $1-2$ hours. He then became aware of persistent numbness and listlessness of the bilateral lower limbs, which gradually interfered with walking. These symptoms aggravated upon walking, which became difficult, but then remitted after stopping and resting for a while, regardless of the posture. The symptoms varied in severity from day to day and sometimes disappeared, but progressed with time to the extent that continuous walking for $\geq 100 \mathrm{~m}$ became difficult. The patient also had occasional difficulty with urination, starting from the same period. He visited a hospital due to gait disorder tending to aggravate and was diagnosed with lumbar spinal canal stenosis due to observation of lumbago and gait disorder, and based on MRI findings. He was treated with spine fusion in addition to laminectomy, but gait disorder due to numbness and listlessness of the bilateral lower limbs did not improve and the patient was referred to our hospital. He had a history of hypertension and diabetes, but no particular family history. He had not smoked cigarettes and only occasionally drank alcohol.

Illness at admission

Numbness and listlessness of the bilateral lower limbs appeared after conti- 
nuous walking for about 1 minute and walking became difficult. Reduced lower limb muscular strength (MMT4) was noted in the right anterior tibial muscle, right extensor pollicis longus, and right flexor pollicis longus. Mild hypoesthesia was observed on the lateral side of the bilateral crura. There was no laterality in the patellar tendon or Achilles tendon reflex (promotion or reduction), and $\mathrm{Ba}$ binski/Chaddock reflexes were negative. A femoral nerve stretch test and a straight leg raising test of the lower limbs were both negative. The bilateral dorsalis pedis arteries were favorably palpated without laterality. The JOA score was 6/29 (I: 3/9, II: 4/6, III: 5/14, IV: -6/-6). No abnormal finding was detected in blood chemistry, and the ankle-brachial index (ABI) was normal in both lower limbs.

Imaging findings

Plain radiography showed that laminectomy of the 5th lumbar vertebra and posterolateral fusion from the 4 th lumbar vertebra to the sacrum had been performed (Figure 1). On spinal CT after myelography, decompression of the lumbar region was favorable, but a worm-like appearance was noted on the dorsal side of the mid-thoracic spine (Figure 2). On thoracolumbar MRI, favorable decompression of the $3 \mathrm{rd}$ and lower lumbar vertebrae had been acquired after surgery, but low T1 and high T2 intensities were detected in the 5th to 12th thoracic vertebra. There was also evidence of abnormal vascular dilatation on the dorsal side of the mid-thoracic spinal region (Figure 3 and Figure 4). Based on these findings, spinal AVM was suspected and selective spinal angiography was performed to make a definite diagnosis. Arterial blood flowed from the radiculomedullary artery of the 7th thoracic spinal nerve into the spinal coronary sinus, causing marked dilation (Figure 5). A shunt was present in the dura and was classified as a spinal dural arteriovenous fistula (AVF) due to spinal AVM. Impaired spinal venous return caused by spinal AVM had induced spinal symptoms, and surgery was performed because the symptoms were progressive.
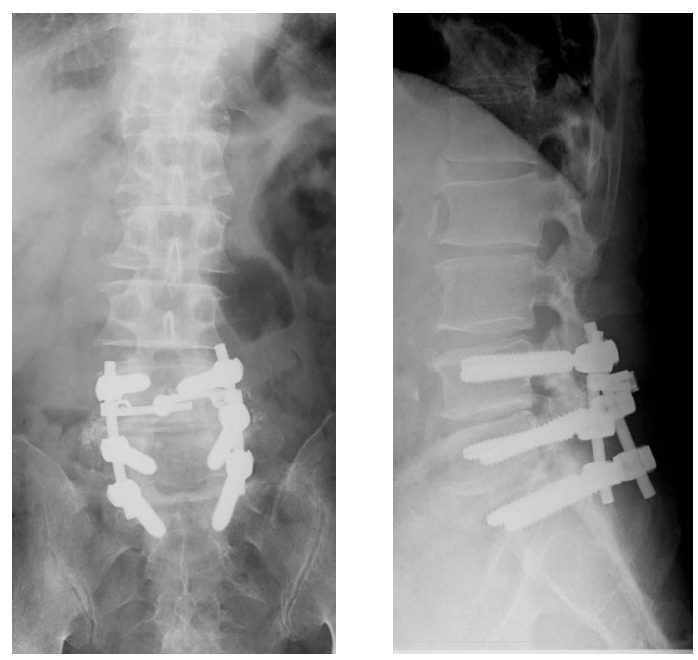

Figure 1. Lumbar radiography. Frontal view (left) and lateral view (right) after laminectomy of the 5th lumbar vertebra and posterolateral fusion from the 4th lumbar vertebra to the sacrum. 

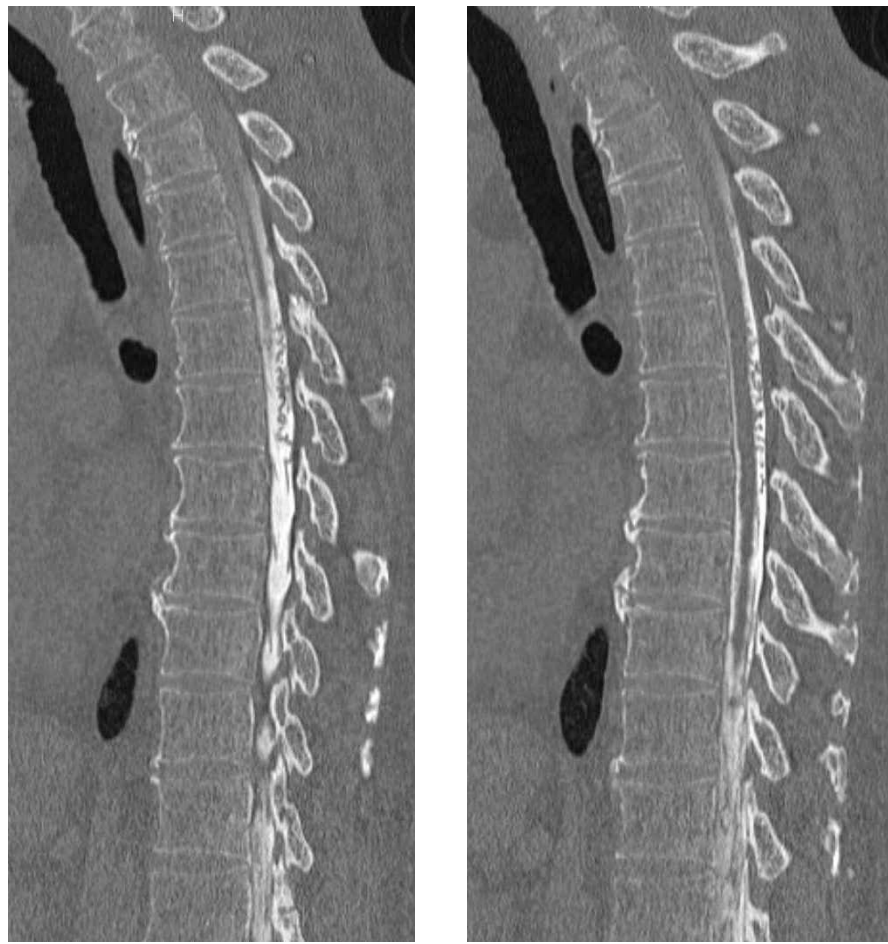

Figure 2. Thoracic spinal CT after myelography (sagittal view). A worm-like appearance was observed on the dorsal side of the spine in the mid-thoracic spinal region.

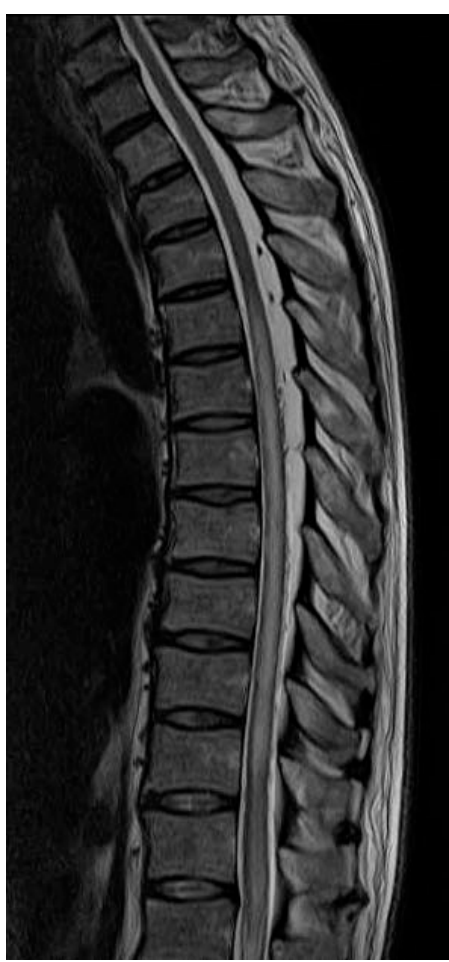

(a)

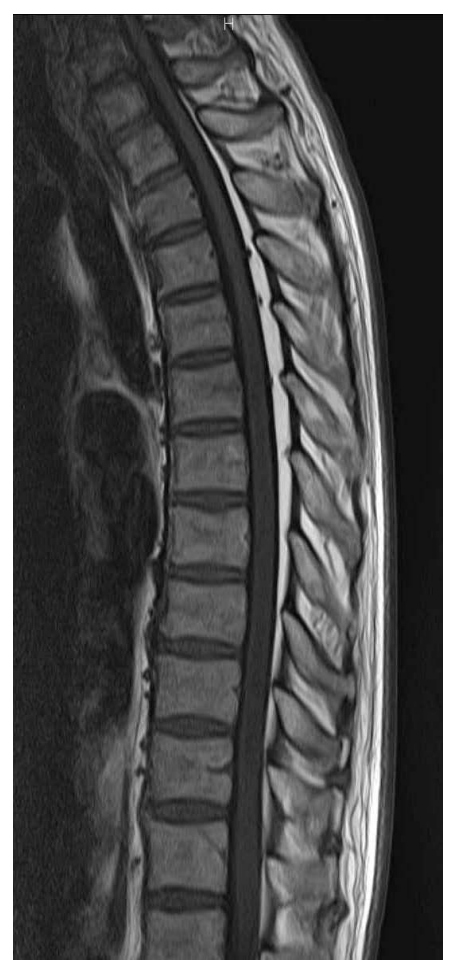

(b)

Figure 3. (a) T2-weighted MRI (sagittal view); (b) T1-weighted MRI (sagittal view). Favorable decompression was acquired at the 3rd lumbar vertebra and lower after surgery, but changes to high $\mathrm{T} 2$ intensity and low $\mathrm{T} 1$ intensity were detected in the spinal cord of the 5 th to 12 th thoracic vertebrae. The subarachnoid space was also dilated. 


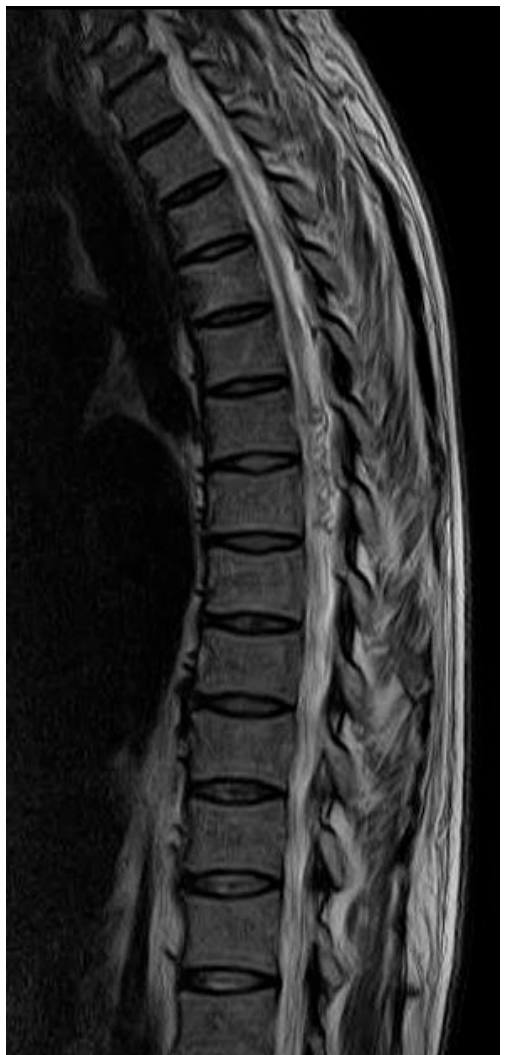

(a)

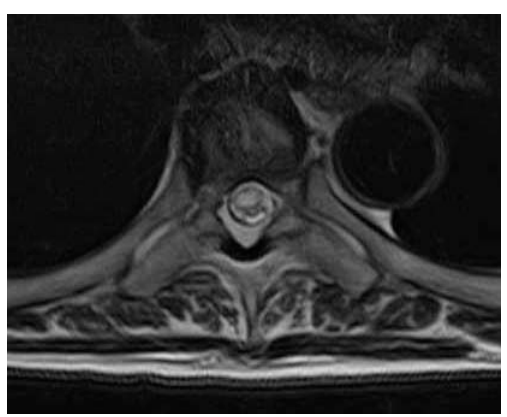

(b)

Figure 4. (a) T2-weighted MRI (sagittal view); (b) T2-weighted MRI (horizontal view). An abnormal dilated blood vessel was observed on the dorsal side of the mid-thoracic spinal region.

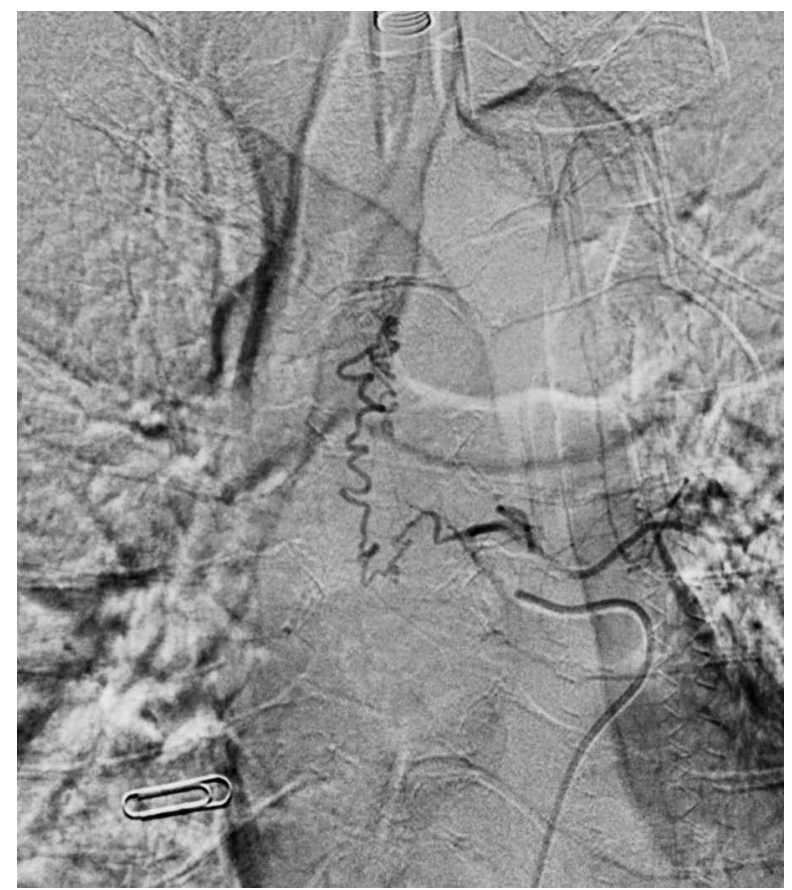

Figure 5. Selective spinal angiography: Arterial blood flowed into the coronary sinus from the radiculomedullary artery of the 7 th thoracic nerve, causing marked dilation. 


\section{Surgical findings}

To confirm the feeder identified by selective spinal angiography, laminectomy of the 6 th to 7 th thoracic vertebrae was performed. The shunt expanding to the superficial layer of the left 7th thoracic nerve root sheath was confirmed and temporarily blocked by clipping. After blocking, the dilated coronary sinus was examined using Doppler ultrasound. Arterial pulses heard before clipping disappeared, the vascular color changed to a venous blood color, and vascular dilation regressed. Ascending and evoked spinal cord potentials induced by electric stimulation of the spinal cord were monitored for 15 minutes after blocking the shunt. An absence of pathological changes in the ascending or descending spinal cord potential was confirmed and the shunt was coagulated and cut.

Postoperative course

Pain and dysuria improved quickly after surgery and intermittent claudication also improved, which allowed the patient to be discharged on postoperative day 16. On MRI at final follow-up 4 year after surgery, there was a tendency for improvement in the thoracic spinal cord high-intensity area detected before surgery (Figure 6). Intermittent claudication had resolved at final follow-up, but mild dysuria remained. The JOA score was 20/29 (I: 7/9, II: 5/6, III: 11/14, IV: $-3 /-6)$, and the improvement rate was $61 \%$.

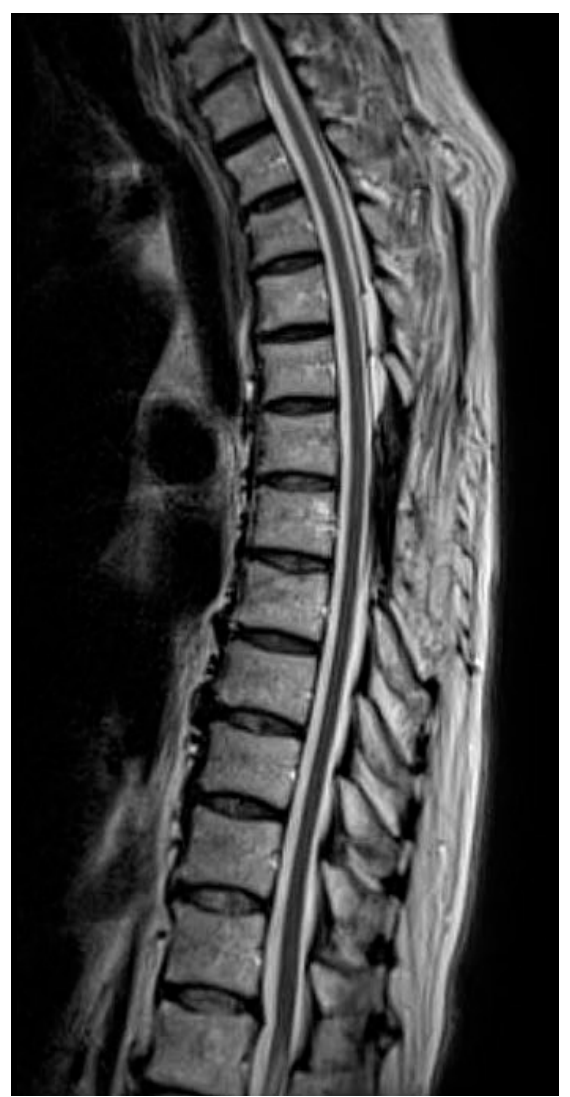

Figure 6. T2-weighted MRI of the thoracic spine at final follow-up (4 years after surgery) (sagittal view). The abnormal blood vessel had disappeared on postoperative MRI, and the high intensity area in the thoracic spinal cord had improved. 


\section{Discussion}

Spinal AVM is defined as the shunt formation by root arteries sending blood flow to the spinal cord and nerve roots, and anterior/posterior spinal cord arteries and veins coming out from the nerve roots and spinal cord. Generally, this condition is considered congenital, but it may be acquired due to inflammation and trauma [1]. Spinal AVM accounts for about $4 \%$ of spinal cord tumor cases and develops more commonly in the lower thoracic than the upper lumbar spine [2]. It is classified based on the development pattern into a rapidly developing apoplectic type; a chronic progressive type, in which symptoms progressively aggravate for a long time after onset; and an intermittent type, in which symptoms develop acutely and repeatedly aggravate and remit over a prolonged period [2]. The disease in our patient may have been the chronic progressive type based on the course. Spinal AVM is also divided into three types based on the shunt location: intramedullary AVM, perimedullary AVF, and dural AVF [3]. Our patient was diagnosed with spinal dural AVF, because shunt formation was observed in the dural region on selective spinal angiography.

The developmental mechanism of the symptoms is thought to be as follows. The spinal venous return is impaired by high-pressure arterial blood flowing into the coronary sinus via a shunt and venous pressure is promoted, which causes spinal cord symptoms to progress gradually [4]. The variation in spinal AVM symptoms is thought to be due to dilation of peripheral blood vessels, which further decreases intramedullary blood flow due to reduced blood pressure and aggravates the ischemic state of the spinal cord [5]. Listlessness, pain, and an abnormal sensation of the lower limb are possible initial symptoms [5], but Abe et al found that fewer patients have disease onset with pain [6]. Pain of the lower limb was also not the initial symptom in our patient. The patient complained of lumbago, but this was improved by body movement and rest, suggesting that it was common lumbago.

Spinal AVM may cause intermittent claudication and bladder and rectal disturbance [1], and differentiation from lumbar degenerative diseases and arteriosclerosis obliterans is required, which may cause difficulty with diagnosis [7]. Intermittent claudication is classified into neurogenic (cauda equina and nerve root), spinal, and arterial types, and each type have characteristic clinical symptoms and properties of claudication [8] (Table 1). In our patient, lower limb pain was absent, but intermittent claudication due to listlessness and numbness of the bilateral lower limbs, reduction of muscular strength of the right toes, and dysuria developed. At an initial glance, this suggests lumbar spinal canal stenosis-induced cauda equina intermittent claudication. However, the symptoms did not improve despite being treated with surgery for spinal canal stenosis by a previous physician. Thus, we strongly suspected spinal intermittent claudication, and we performed a spinal CT and MRI after myelography for the entire spinal cord, and identified the lesion in the thoracic spinal cord. MRI is useful for screening for spinal AVM [7]. Characteristic MRI findings include changes to a 
high intramedullary intensity on T2-weighted imaging and spinal cord enlargement, which are considered to be due to circulatory disorder-induced edema [9]. In addition, an emissary vein distributing in the sagittal direction while meandering on the spinal cord surface is visualized as an abnormal blood vessel on T2-weighted imaging, with a frequency of $35 \%$ to $<50 \%$ [7].

The objective of treatment of spinal dural AVF is to occlude the shunt, for which endovascular and surgical treatments can be used. Embolization using n-butyl-2-cyanoacrylate (NBCA) gives favorable treatment outcomes, but Guillevin et al. found a recurrence rate after embolization of about 30\% [10] and Okuda et al. mentioned a risk of NBCA flowing into the anterior spinal cord artery [11]. Bakker et al. found a higher treatment success rate and lower recurrence rate with surgical treatment compared to embolization [12] (Table 2), but complications of surgical treatment can also occur. Thus, Huffmann et al. reported postoperative complications of cerebrospinal fluid leakage, delay in wound healing, dural hematoma, and venous thrombosis, although these developed in only a few cases [13]. To ensure closing the shunt under direct vision, we selected surgery in our case.

The postoperative outcome and prognosis of spinal dural AVF are not influenced by age, sex, and location of the lesion [14], but early diagnosis and early treatment have been proposed as prognostic factors because the postoperative

Table 1. Classification and characteristics of intermittent claudication.

\begin{tabular}{|c|c|c|c|c|}
\hline & \multicolumn{2}{|c|}{ Neurogenic } & \multirow{2}{*}{$\begin{array}{c}\text { Spinal } \\
\text { cord-induced }\end{array}$} & \multirow{2}{*}{ Arterial } \\
\hline & Cauda equina & Nerve root & & \\
\hline Induction & $\begin{array}{l}\text { Walking } \\
\text { (with postural } \\
\text { factor) }\end{array}$ & $\begin{array}{l}\text { Walking } \\
\text { (with postural } \\
\text { factor) }\end{array}$ & $\begin{array}{c}\text { Walking } \\
\text { (without } \\
\text { postural factor) }\end{array}$ & $\begin{array}{c}\text { Walking } \\
\text { (without } \\
\text { postural factor) }\end{array}$ \\
\hline Symptoms & $\begin{array}{l}\text { Abnormal sensation, } \\
\text { such as numbness } \\
\text { and cold sense }\end{array}$ & Pain & $\begin{array}{c}\text { Abnormal } \\
\text { sensation, such } \\
\text { as heaviness } \\
\text { and dullness, } \\
\text { not typicalpain }\end{array}$ & Pain \\
\hline $\begin{array}{l}\text { Location of } \\
\text { symptoms }\end{array}$ & $\begin{array}{c}\text { Posterior surface } \\
\text { of the lower limb, } \\
\text { bilateral }\end{array}$ & $\begin{array}{l}\text { Posterior surface } \\
\text { of the lower limb, } \\
\text { unilateral }\end{array}$ & $\begin{array}{c}\text { Entire } \\
\text { lower limb, } \\
\text { bilateral }\end{array}$ & $\begin{array}{l}\text { Posterior surface } \\
\text { of the crus over } \\
\text { the foot, unilateral }\end{array}$ \\
\hline Arterial pulse & Normal & Normal & Normal & Lost or weakened \\
\hline
\end{tabular}

Table 2. Comparison of treatment outcomes of spinal dural arteriovenous fistulausing surgical treatment and embolization.

\begin{tabular}{lccc}
\hline & First treatment Success rate & Recurrence rate & Serious complication \\
\hline Surgical treatment & $96.6 \%$ & $2.3 \%$ & $0 \%$ \\
Embolization & $72.2 \%$ & $45.5 \%$ (Onyx) & $2 \%$ \\
& & $23.0 \%(\mathrm{NBCA})^{*}$ & $($ spinal cord infarction) \\
\hline
\end{tabular}

*NBCA: n-butyl-2-cyanoacrylate. 
outcome is poor in patients with high preoperative severity and a long duration of illness [5]. In our patient, the preoperative JOA score was 6, showing high preoperative severity, and the duration of illness was 1.5 years before diagnosis, which may explain the limited improvement of the JOA score to 20 at final follow-up. In a case with these characteristics, we suggest that close examination of the entire spinal cord is needed for effective treatment.

\section{Conclusion}

We encountered a patient with spinal AVM-induced intermittent claudication. A "worm-like appearance" on CT after myelography that is a characteristic of spinal AVM permitted diagnosis, but marked improvement could not be achieved because of the prolonged course from onset to diagnosis. In such a case, in which intermittent claudication is difficult to treat, a close examination of the entire spinal cord is recommended to determine the origin of the disease.

\section{Informed Consent}

We obtained the informed consent from the patient to report this case.

\section{Conflicts of Interest}

The authors declare no conflicts of interest regarding the publication of this paper.

\section{References}

[1] Symon, L., Kuyama, H. and Kendall, B. (1984) Dral Arteriovenous Malformations of the Spine. Clinical Features and Surgical Results in 55 Cases. Journal of Neurosurgery, 60, 238-247. https://doi.org/10.3171/jns.1984.60.2.0238

[2] Pia, H.W. and Djindjian, R. (1978) Spinal Angiomas, Advances in Diagnosis and Therapy. Springer-Verlag, Berlin. https://doi.org/10.1007/978-3-642-66720-6

[3] Mourier, K.L., Gobin, Y.P., George, B., Lot, G. and Merland, J.J. (1993) Intradural Perimedullary Arteriovenous Fistulae: Result of Surgical and Endovascular Treatment in Aseries of 35 Cases. Neurosurgey, 32, 885-891. https://doi.org/10.1227/00006123-199306000-00001

[4] Criscuolo, G.R., Oldfield, E.H. and Doppman, J.L. (1989) Reversible Acute and Subacute Myelopathy in Patients with Dural Arteriovenous Fistulas. Foix-Alajouanine Syndrome Reconsidered. Journal of Neurosurgery, 70, 354-359. https://doi.org/10.3171/jns.1989.70.3.0354

[5] Behrens, S. and Thron, A. (1999) Long-Term Follow-Up and Outcome in Patients Treated for Spinal Dural Arteriovenous Fistula. Journal of Neurosurgery, 246, 181-185. https://doi.org/10.1007/s004150050331

[6] Abe, H., et al. (1995) Surgery the Spinal Cord. 256-268.

[7] Gilbertson, J.R., Miller, G.M., Goldman, M.S. and Marsh, W.R. (1995) Spinal Dural Arteriovenous Fistulas: MR and Myelographic Findings. American Journal of Neuroradiology, 16, 2049-2057.

[8] Baba, H., Nakahashi, K., et al. (1990) Clinical Experience of Spinal Intermittent Claudication. The Spinal Cord, 3, 807-812. 
[9] Isu, T., Iwasaki, Y., Akino, M., Koyanagi, I. and Abe, H. (1989) Magnetic Resonance Imaging in Cases of Spinal Dural Arteriovenous Malformation. Neurosurgy, 24, 919-923. https://doi.org/10.1227/00006123-198906000-00023

[10] Guillevin, R., Vallee, J.N., Cormier, E., Lo, D., Dormont, D. and Chiras, J. (2005) N-Butyl 2-Cyanoacrylate Embolization of Spinal Dural Arteriovenous Fistulae: CT Evaluation, Technical Features, and Outcome Prognosis in 26 Cases. American Journal of Neuroradiology, 26, 929-935.

[11] Okuda, A., et al. (2002) Diagnosis and Treatment of the Spinal Corddural Arteriovenous Fistula. The Journal of the Chugoku-Shikoku Orthopedic Association, 14, 171-180.

[12] Bakker, N.A., Uyttenboogaart, M., Luijckx, G.J., et al. (2015) Recurrence Rates after Surgical or Endovascular Treatment of Spinal Dural Arteriovenous Fistulas: A Meta-Analysis. Neurosurgery, 77, 137-144. https://doi.org/10.1227/NEU.0000000000000727

[13] Huffmann, B.C., Gilsbach, J.M. and Thron, A. (1995) Spinal Dural Arteriovenous Fistulas: A Plea for Neurosurgical Treatment. Acta Neurochirurgica, 135, 44-51. https://doi.org/10.1007/BF02307413

[14] Zogopoulos, P., Nakamura, H., Ozaki, T., et al. (2016) Endovascular and Surgical Treatment of Spinal Dural Arteriovenous Fistulas: Assessment of Post-Treatment Clinical Outcome. Neurologia Medico-Chirurgica (Tokyo), 56, 27-32. 